\title{
A Note on Continuity of Solution Set for Parametric Weak Vector Equilibrium Problems
}

\author{
Pakkapon Preechasilp $^{1}$ and Rabian Wangkeeree ${ }^{2}$ \\ ${ }^{1}$ Program in Mathematics, Faculty of Education, Pibulsongkram Rajabhat University, Phitsanulok 65000, Thailand \\ ${ }^{2}$ Department of Mathematics, Faculty of Science, Naresuan University, Phitsanulok 65000, Thailand \\ Correspondence should be addressed to Pakkapon Preechasilp; preechasilpp@gmail.com
}

Received 31 August 2014; Revised 22 December 2014; Accepted 11 January 2015

Academic Editor: Yunhai Xiao

Copyright (c) 2015 P. Preechasilp and R. Wangkeeree. This is an open access article distributed under the Creative Commons Attribution License, which permits unrestricted use, distribution, and reproduction in any medium, provided the original work is properly cited.

\begin{abstract}
We consider the parametric weak vector equilibrium problem. By using a weaker assumption of Peng and Chang (2014), the sufficient conditions for continuity of the solution mappings to a parametric weak vector equilibrium problem are established. Examples are provided to illustrate the essentialness of imposed assumptions. As advantages of the results, we derive the continuity of solution mappings for vector optimization problems.
\end{abstract}

\section{Introduction}

It is well known that the vector equilibrium problem provides a unified model of several classes of problems, including vector variational inequality problems, vector complementarity problems, vector optimization problems, and vector saddle point problems. There are many papers which have discussed the existence results for different types of vector equilibrium problems (see [1-3] and references therein).

In 2008 Gong [4] studied parametric vector equilibrium problems. Based on a scalarization representation of the solution mapping and the property involving the union of a family of lower semicontinuous set-valued mappings of Cheng and Zhu [5], they established the sufficient conditions for the continuity of the solution set mapping for the mixed parametric monotone weak vector equilibrium problems in topological vector spaces. In the same year, Gong and Yao [6] discussed the lower semicontinuity of the efficient solution mappings to a parametric strong vector equilibrium problem with $C$-strict monotonicity of a vector-valued function, by using a scalarization method and density result. In 2009, Xu and $\mathrm{Li}$ [7] presented a new proof of lower semicontinuity of the set of efficient solutions to a parametric strong vector equilibrium problem, which is different from the one used in [6]. In 2010, Chen and $\mathrm{Li}$ [8] discussed and improved the lower semicontinuity and continuity results of efficient solution mappings to a parametric strong vector equilibrium problem in $[4,6]$, without the uniform compactness assumption. By virtue of the scalarization technique, [4, 68 ] have discussed the lower semicontinuity, in the case that $\xi$-efficient solution set is a singleton. However, in practical, the $\xi$-solution set may not be singleton but a general set. Recently, by using a weak assumption, Peng and Chang [9] discussed the lower semicontinuity of solution maps for parametric weak vector equilibrium problem under the case that the $\xi$-efficient solution mapping may not be singlevalued as follows. Unfortunately, the results obtained in the corresponding papers [4,6-9] cannot be used in the case of vector optimization problems. Hence, in this paper, we study the lower semicontinuity of the set of efficient solutions for parametric weak vector equilibrium problems when the $\xi$ efficient solution set is a general set. Moreover, our theorems can apply for vector optimization problems.

The structure of the paper is as follows. Section 2 presents the efficient solutions to parametric weak vector equilibrium problems and materials used in the rest of this paper. We establish, in Section 3, a sufficient condition for the continuity of the efficient solution mappings. We give some examples to illustrate that our main results are different from the corresponding ones in the literature. Section 4 is reserved 
for an application of the main result to a weak vector optimization problem.

\section{Preliminaries}

Throughout this paper, if not otherwise specified, $X, Y$ will denote two real Hausdorff topological vector spaces, and $Z$ a real topological space, and $M$ a nonempty subset of $Z$. Let $Y^{*}$ be the topological dual space of $Y$. Let $C \subset Y$ be a pointed, closed, and convex cone with int $C \neq \emptyset$. Let

$$
C^{*}:=\left\{\xi \in Y^{*}: \xi(y) \geq 0, \forall y \in C\right\}
$$

be the dual cone of $C$. Denote the quasi-interior of $C^{*}$ by $C^{\natural}$; that is,

$$
C^{\natural}:=\left\{\xi \in Y^{*}: \xi(y)>0, \forall y \in C \backslash\{0\}\right\} .
$$

Since int $C \neq \emptyset$, the dual cone $C^{*}$ of $C$ has a weak ${ }^{*}$ compact base. Let $e \in \operatorname{int} C$. Then,

$$
B_{e}^{*}:=\left\{\xi \in C^{*}: \xi(e)=1\right\}
$$

is a weak ${ }^{*}$ compact base of $C^{*}$.

Let $N\left(\mu_{0}\right) \subset M$ be neighborhoods of considered points $\mu_{0}$. Let $A: M \rightrightarrows X$ be a set-valued mapping and let $f$ : $X \times X \times M \rightarrow Y$ be a vector-valued mapping.

For each $\mu \in N\left(\mu_{0}\right)$, we consider the following parametric weak vector equilibrium problem (PWVEP): find $x \in A(\mu)$, such that

$$
f(x, y, \mu) \notin-\operatorname{int} C, \quad \forall y \in A(\mu) .
$$

Let $S(\mu)$ be the efficient solution set of $(4)$; that is,

$$
S(\mu):=\{x \in A(\mu): f(x, y, \mu) \notin-\operatorname{int} C, \forall y \in A(\mu)\} .
$$

For each $\xi \in C^{*} \backslash\{0\}$ and $\mu \in N\left(\mu_{0}\right)$, let $S_{\xi}(\mu)$ denote the set of $\xi$-efficient solution set to (4); that is,

$$
S_{\xi}(\mu):=\{x \in A(\mu): \xi(f(x, y, \mu)) \geq 0, \forall y \in A(\mu)\} .
$$

Throughout this paper, we always assume $S(\mu) \neq \emptyset$ for all $\mu \in \Lambda$. Now, we recall the definition of semicontinuity of setvalued mappings. Let $\Lambda$ and $X$ be two topological spaces, $F$ : $\Lambda \rightarrow 2^{X}$ a set-valued mapping, and $\bar{\lambda} \in \Lambda$.

Definition 1 (see [10]). (i) $F$ is said to be lower semicontinuous (l.s.c.) at $\bar{\lambda}$ if, for any open set $U$ satisfying $U \cap F(\bar{\lambda}) \neq \emptyset$, there exists $\delta>0$ such that $F(\lambda) \cap U \neq \emptyset$, for all $\lambda \in B(\bar{\lambda}, \delta)$.

(ii) $F$ is said to be upper semicontinuous (u.s.c) at $\bar{\lambda}$ if, for any open set $U$ satisfying $F(\bar{\lambda}) \subset U$, there exists $\delta>0$ such that $F(\lambda) \subset U$, for all $\lambda \in B(\bar{\lambda}, \delta)$.

Proposition 2 (see $[11,12]$ ). (i) $F$ is l.s.c. at $\bar{\lambda}$ if and only if, for any net $\left\{\lambda_{\alpha}\right\} \subset \Lambda$ with $\lambda_{\alpha} \rightarrow \bar{\lambda}$ and any $\bar{x} \in F(\bar{\lambda})$, there exists $x_{\alpha} \in F\left(\lambda_{\alpha}\right)$ such that $x_{\alpha} \rightarrow \bar{x}$.

(ii) If $F$ has compact values (i.e., $F(\lambda)$ is a compact set for each $\lambda \in \Lambda)$, then $F$ is u.s.c. at $\bar{\lambda}$ if and only if, for any net $\left\{\lambda_{\alpha}\right\} \subset \Lambda$ with $\lambda_{\alpha} \rightarrow \bar{\lambda}$ and for any $x_{\alpha} \in F\left(\lambda_{\alpha}\right)$, there exists $\bar{x} \in F(\bar{\lambda})$ and a subnet $\left\{x_{\beta}\right\}$ of $\left\{x_{\alpha}\right\}$ such that $x_{\beta} \rightarrow \bar{x}$.
Definition 3. Let $X$ and $Y$ be two vector spaces. Let $B$ be a nonempty subset of $X$. A vector-valued function $\varphi: B \rightarrow Y$ is said to be

(a) C-strictly convex on a convex subset $K$ of $B$, if

$$
\begin{array}{r}
t \varphi(x)+(1-t) \varphi(y) \in \varphi(t x+(1-t) y)+\operatorname{int} C, \\
\forall x, y \in K \quad \text { with } x \neq y, \quad \forall t \in(0,1) ;
\end{array}
$$

(b) $C$-convex on a convex subset $K$ of $B$, if

$$
\begin{array}{r}
t \varphi(x)+(1-t) \varphi(y) \in \varphi(t x+(1-t) y)+C, \\
\forall x, y \in K, \quad \forall t \in[0,1] ;
\end{array}
$$

(c) C-convexlike on convex subset $K$ of $B$, if, for any $x_{1}$, $x_{2} \in K$ and any $t \in[0,1]$, there exist $x_{3} \in K$ such that $t f\left(x, x_{1}, \mu\right)+(1-t) f\left(x, x_{2}, \mu\right) \in f\left(x, x_{3}, \mu\right)+C$.

Obviously, we get that

$$
(a) \Longrightarrow(b) \Longrightarrow(c) \text {. }
$$

Next, we recall the definitions of monotonicity which are in common use in review literature.

Definition 4. Let $X$ and $Y$ be two vector spaces. Let $B$ be a nonempty subset of $X$. A bifunction $f: B \times B \rightarrow Y$ is said to be

(i) monotone on subset $K$ of $B$, if

$$
f(x, y)+f(y, x) \in-C, \quad \forall x, y \in K
$$

(ii) strictly monotone on subset $K$ of $B$, if $f$ is monotone and

$$
f(x, y)+f(y, x) \in-\operatorname{int} C, \quad \forall x, y \in K, x \neq y .
$$

Remark 5. It is clear that (ii) implies (i) but the converse is not true. An easy example is that $f(x, y)=g(y)-g(x)$ for all $x, y \in B$ where $g: B \rightarrow Y$; we see that $f(x, y)+f(y, x)=$ $(g(y)-g(x))+(g(x)-g(y))=0_{Y} \notin-\operatorname{int} C$ for all $x, y \in K$.

Now, we collect two vital lemmas.

Lemma 6 (see [13]). Suppose that for each $\mu \in M$ and $x \in$ $A(\mu), f(x, A(\mu), \mu)+C$ is a convex set; then

$$
S(\mu)=\bigcup_{\xi \in C^{*} \backslash\{0\}} S_{\xi}(\mu)
$$

Lemma 7 (see [14, Theorem 2, p. 114]). The union $\Gamma=\cup_{i \in I} \Gamma_{i}$ of a family of l.s.c. set-valued mappings $\Gamma_{i}$ from a topological space $X$ to a topological space $Y$ is also l.s.c. set-valued mapping from $X$ to $Y$ is also l.s.c. set-valued mapping from $X$ to $Y$, where $I$ is an index set. 


\section{Main Results}

In this section, we present the continuity of the efficient solution mapping to PWVEP.

Theorem 8. Let $\mu_{0} \in M$ be a considered point for (PWVEP). Suppose that the following conditions are satisfied:

(i) $A(\cdot)$ is continuous with nonempty compact convex values at $\mu_{0}$;

(ii) $f(\cdot, \cdot, \cdot)$ is continuous on $B \times B \times M$;

(iii) $f\left(\cdot, \cdot, \mu_{0}\right)$ is monotone on $A\left(\mu_{0}\right)$;

(iv) $f\left(x, \cdot, \mu_{0}\right)$ is $C$-strictly convex on $A\left(\mu_{0}\right)$.

Then, for each $\xi \in C^{*} \backslash\{0\}, S_{\xi}(\cdot)$ is continuous on $\mu_{0}$.

Proof. We first prove that $S(\cdot)$ is lower semicontinuous at $\mu_{0}$. Suppose the contrary that there exists a $\xi^{\prime} \in C^{*} \backslash\{0\}$ such that $S_{\xi^{\prime}}(\cdot)$ is not l.s.c. at $\mu_{0}$. Then there exists a net $\left\{\mu_{\alpha}\right\}$ with $\mu_{\alpha} \rightarrow \mu_{0}$ and $x_{0} \in S\left(\mu_{0}\right)$ such that for any $x_{\alpha} \in S_{\xi^{\prime}}\left(\mu_{\alpha}\right)$, $x_{\alpha} \nrightarrow x_{0}$. Since $x_{0} \in S_{\xi^{\prime}}\left(\mu_{0}\right)$, we have $x_{0} \in A\left(\mu_{0}\right)$ and

$$
\xi^{\prime}\left(f\left(x_{0}, y, \mu_{0}\right)\right) \geq 0, \quad \forall y \in A\left(\mu_{0}\right) .
$$

By the lower semicontinuity of $A(\cdot)$ at $\mu_{0}$, there exists a net $\left\{\bar{x}_{\alpha}\right\} \subset A\left(\mu_{\alpha}\right)$ such that $\bar{x}_{\alpha} \rightarrow x_{0}$.

For any $y_{\alpha} \in S\left(\mu_{\alpha}\right)$, by the upper semicontinuity and compactness of $A(\cdot)$ at $\mu_{0}$, we get that there exists $y_{0} \in A\left(\mu_{0}\right)$ and a subsequence $\left\{y_{\alpha_{i}}\right\}$ of $\left\{y_{\alpha}\right\}$ such that $y_{\alpha_{i}} \rightarrow y_{0}$, denoted by $\left\{y_{i}\right\}$. We have

$$
\xi^{\prime}\left(f\left(y_{i}, x_{i}, \mu_{i}\right)\right) \geq 0 \quad \forall i, \xi^{\prime}\left(f\left(x_{0}, y_{0}, \mu_{0}\right)\right) \geq 0 .
$$

By continuity of $\xi^{\prime}$ and $f(\cdot, \cdot, \cdot)$ on $B \times B \times M$, we get that

$$
\xi^{\prime}\left(f\left(y_{0}, x_{0}, \mu_{0}\right)\right) \geq 0 .
$$

We want to show that $x_{0}=y_{0}$. Assume that $x_{0} \neq y_{0}$, then by strict convexity of $f\left(x, \cdot, \mu_{0}\right)$ and linearity of $\xi^{\prime}$ imply that

$$
\begin{aligned}
0 & \leq \xi^{\prime}\left(f\left(x_{0}, \frac{1}{2} x_{0}+\frac{1}{2} y_{0}, \mu_{0}\right)\right) \\
& <\frac{1}{2} \xi^{\prime}\left(f\left(x_{0}, y_{0}, \mu_{0}\right)\right)+\frac{1}{2} \xi^{\prime}\left(f\left(y_{0}, x_{0}, \mu_{0}\right)\right), \\
0 & \leq \xi^{\prime}\left(f\left(y_{0}, \frac{1}{2} x_{0}+\frac{1}{2} y_{0}, \mu_{0}\right)\right) \\
& <\frac{1}{2} \xi^{\prime}\left(f\left(y_{0}, x_{0}, \mu_{0}\right)\right)+\frac{1}{2} \xi^{\prime}\left(f\left(y_{0}, y_{0}, \mu_{0}\right)\right) .
\end{aligned}
$$

Monotonicity assumption of $f\left(\cdot, \cdot, \mu_{0}\right)$ implies that

$$
\begin{aligned}
0 & <\frac{1}{2} \xi^{\prime}\left(f\left(x_{0}, x_{0}, \mu_{0}\right)\right)+\frac{1}{2} \xi^{\prime}\left(f\left(y_{0}, x_{0}, \mu_{0}\right)\right) \\
& \leq \frac{1}{2} \xi^{\prime}\left(f\left(y_{0}, x_{0}, \mu_{0}\right)\right), \\
0 & <\frac{1}{2} \xi^{\prime}\left(f\left(y_{0}, x_{0}, \mu_{0}\right)\right)+\frac{1}{2} \xi^{\prime}\left(f\left(y_{0}, y_{0}, \mu_{0}\right)\right) \\
& \leq \frac{1}{2} \xi^{\prime}\left(f\left(y_{0}, x_{0}, \mu_{0}\right)\right) .
\end{aligned}
$$

This implies that

$$
\begin{aligned}
& 0<\xi^{\prime}\left(f\left(y_{0}, x_{0}, \mu_{0}\right)\right), \\
& 0<\xi^{\prime}\left(f\left(y_{0}, x_{0}, \mu_{0}\right)\right) .
\end{aligned}
$$

Adding (18) and (19), it follows from linearity of $\xi^{\prime}$ and monotonicity of $f$ that

$$
0<\xi^{\prime}\left(f\left(x_{0}, y_{0}, \mu_{0}\right)+f\left(y_{0}, x_{0}, \mu_{0}\right)\right) \leq 0 .
$$

This is impossible by the contradiction assumption. This proof is complete.

Before comparing our result with the result of [9], we first recall that result as follows.

Theorem 9 (see [9, Theorem 3.1]). Let $\mu_{0} \in M$ be a considered point for (PWVEP). Suppose that the following conditions are satisfied:

(i) $A(\cdot)$ is a mapping with nonempty compact convex valued and continuous at $\mu_{0}$;

(ii) for each $\mu \in M,(x, y) \mapsto f(x, y, \mu)$ is continuous on $B \times B$;

(iii) for any $x, y \in A\left(\mu_{0}\right)$, if $x \neq y$, then $f\left(x, y, \mu_{0}\right)+$ $f\left(y, x, \mu_{0}\right) \in-\operatorname{int} C$.

Then, for each $\xi \in C^{*} \backslash\{0\}, S_{\xi}(\cdot)$ is l.s.c. at $\mu_{0}$.

Remark 10. In [9], they assumed the condition of $C$-strict monotonicity (or called $C$-strongly monotone in $[6,7]$ ) at the considered point $\mu_{0}$. In the case, the $\xi$-solution set may be a general set, but not a singleton. Unfortunately, that result of [9] cannot be used in the case of vector optimization problems. Theorem 8 discusses the lower semicontinuity of the $\xi$-solution mappings. Compared with Theorem 3.1 of [9], assumption (iii) of Theorem 8 is relaxed from assumption (iii) in Theorem 3.1 in [9]. An advantage Theorem 8 is that it works for vector optimization problems. However, in some situations Theorem 8 is applicable while Theorem 3.1 in [9] is not, as shown by the following example.

Example 11. Let $X=Z=\mathbb{R}, Y=\mathbb{R}^{2}, C=\mathbb{R}_{+}^{2}, M=[1,2]$ be a subset of $Z$. Let $\mu_{0}=1 \in M$ be a considered point for (PVEP). Let $A: M \rightarrow X$ be a mapping defined by $A(\mu)=[1,2]$ and let $f: X \times X \times M \rightarrow Y$ be a mapping defined by

$$
f(x, y, \mu)=\left(x\left(\mu y^{2}-x^{2}\right), \mu\left(y^{2}-x^{2}\right)\right) .
$$

It is clear that $f$ is monotone on $A\left(\mu_{0}\right)$, but not satisfied condition (iii) in Theorem 3.1 of [9]. Indeed, for each $x, y \in$ $A\left(\mu_{0}\right)=[1,2]$, we have

$$
\begin{aligned}
f\left(x, y, \mu_{0}\right)+f\left(y, x, \mu_{0}\right) \\
=\left(x\left(y^{2}-x^{2}\right), y^{2}-x^{2}\right)+\left(y\left(x^{2}-y^{2}\right), x^{2}-y^{2}\right) \\
=\left(-\left(x^{3}-x^{2} y-x y^{2}+y^{3}\right), 0\right) .
\end{aligned}
$$


Also, $f\left(x, \cdot, \mu_{0}\right)$ satisfy $C$-strictly convex on $A\left(\mu_{0}\right)$. Indeed, for any $t \in(0,1)$ and $y_{1}, y_{2} \in A\left(\mu_{0}\right)$, we have

$$
\begin{aligned}
t f\left(x, y_{1}, \mu_{0}\right)+(1-t) f\left(x, y_{2}, \mu_{0}\right) \\
-f\left(x, t y_{1}+(1-t) y_{2}, \mu_{0}\right) \\
=t\left(x\left(y_{1}^{2}-x^{2}\right), y_{1}^{2}-x^{2}\right) \\
\quad+(1-t)\left(x\left(y_{2}^{2}-x^{2}\right), y_{2}^{2}-x^{2}\right) \\
\quad-\left(x\left(\left(t y_{1}+(1-t) y_{2}\right)^{2}-x^{2}\right)\right. \\
\left.\quad\left(t y_{1}+(1-t) y_{2}\right)^{2}-x^{2}\right) \\
=\left(x\left(t y_{1}^{2}+(1-t) y_{2}^{2}\right)-x\left(t y_{1}+(1-t) y_{2}\right)^{2}\right. \\
\left.t y_{1}^{2}+(1-t) y_{2}^{2}-\left(t y_{1}+(1-t) y_{2}\right)^{2}\right) \in \operatorname{int} C .
\end{aligned}
$$

Let $\bar{\xi}=(1,0) \in C^{*} \backslash\{0\}$. We directly compute that $S_{\bar{\xi}}(\mu)=$ $[1, \sqrt{\mu}]$, for each $\mu \in M$. Thus, we can easily get that $S_{\bar{\xi}}\left(\mu^{\prime}\right)=$ $\left[1, \sqrt{\mu^{\prime}}\right]$ is a general set-valued one for each $\mu^{\prime} \in N\left(\mu_{0}\right) \cap$ $M \backslash\left\{\mu_{0}\right\}$ (where $N\left(\mu_{0}\right)$ is any neighborhood of $\mu_{0}$ ), but not a singleton. Moreover, by Theorem 8 , we can get that $S_{\bar{\xi}}(\cdot)$ is l.s.c. at $\mu_{0}$.

However, to relax the condition (iii) in [9], we add the condition of strict convexity of $f$. The following example illustrates that the strict convexity of $f$ is needed.

Example 12. Let $X=Z=\mathbb{R}, Y=\mathbb{R}^{2}, C=\mathbb{R}_{+}^{2}, M=[0,1]$ be a subset of $Z$. Let $\mu_{0}=1 \in M$ be a considered point for (PVEP). Let $A: M \rightarrow X$ be a mapping defined by $A(\mu)=[0,1]$ and let $f: X \times X \times M \rightarrow Y$ be a mapping defined by

$$
f(x, y, \mu)=(\mu x(y-x), \mu(y-x)) .
$$

It is clear that $f$ is monotone on $A\left(\mu_{0}\right)$ and $f\left(x, \cdot, \mu_{0}\right)$ also does not satisfy $C$-strictly convex on $A\left(\mu_{0}\right)$. Let $\xi=(1,0) \in C^{*} \backslash\{0\}$. It follows from direct computation that

$$
S(\mu)= \begin{cases}{[0,1],} & \text { if } \mu=0, \\ \{0\}, & \text { if } \mu \in(0,1] .\end{cases}
$$

Clearly, we see that $S(\cdot)$ is not l.s.c. at $\mu_{0}$. Hence, the assumed strict convexity of $f$ is essential.

Theorem 13. Let $\mu_{0} \in M$ be a considered point. Suppose that the following conditions are satisfied:

(i) $A(\cdot)$ is continuous with nonempty compact convex values at $\mu_{0}$;

(ii) $f(\cdot, \cdot, \cdot)$ is continuous on $B \times B \times M$;

(iii) $f\left(\cdot, \cdot, \mu_{0}\right)$ is monotone on $A\left(\mu_{0}\right)$;

(iv) for each $x \in A\left(\mu_{0}\right), f\left(x, \cdot, \mu_{0}\right)$ is C-strictly convex on $A\left(\mu_{0}\right)$; (v) for each $\mu \in M$ and $x \in A(\mu), f(x, \cdot, \mu)$ is $C$-convexlike on $A(\mu)$.

Then, $S(\cdot)$ is l.s.c. at $\mu_{0}$.

Proof. Since, for each $\mu \in M$ and for each $x \in A(\mu), f(x, \cdot, \mu)$ is $C$-convexlike on $A(\mu), F(x, A(\mu), \mu)+C$ is convex. It follows from Lemma 6 that

$$
S(\mu)=\bigcup_{\xi \in C^{*} \backslash\{0\}} S_{\xi}(\mu) .
$$

By Theorem 8, for each $\xi \in C^{*} \backslash\left\{0_{Y^{*}}\right\}, S_{\xi}(\cdot)$ is 1.s.c. at $\mu_{0}$. Therefore, by Lemma 7 it implies that $S(\cdot)$ is l.s.c. at $\mu_{0}$. This completes the proof.

Now, we give an example to illustrate that our result improves that of [9].

Example 14. Let $X=Z=\mathbb{R}, Y=\mathbb{R}^{2}, C=\mathbb{R}_{+}^{2}, M=[1,2]$ be a subset of $Z$. Let $\mu_{0}=1 \in M$ be a considered point for (PVEP). Let $A: M \rightarrow X$ be a mapping defined by $A(\mu)=[\mu, 6]$ and let $f: X \times X \times M \rightarrow Y$ be a mapping defined by

$$
f(x, y, \mu)=\left(x\left(\mu y^{2}-x^{2}\right), \mu\left(y^{2}-x^{2}\right)\right) .
$$

It is clear that $f$ is monotone on $A\left(\mu_{0}\right)$, but not satisfied $C$ strict monotone on $A\left(\mu_{0}\right)$. Also, $f\left(x, \cdot, \mu_{0}\right)$ satisfy $C$-strictly convex on $A\left(\mu_{0}\right)$. It follows from direct computation that $S(\mu)=[\mu, \mu \sqrt{\mu}]$, for each $\mu \in M$. Thus, we can easily get that $S_{\bar{\xi}}\left(\mu^{\prime}\right)=\left[\mu^{\prime}, \mu^{\prime} \sqrt{\mu^{\prime}}\right]$ is a general set-valued one for each $\mu^{\prime} \in N\left(\mu_{0}\right) \cap M \backslash\left\{\mu_{0}\right\}$ (where $N\left(\mu_{0}\right)$ is any neighborhood of $\left.\mu_{0}\right)$, but not a singleton. Moreover, by Theorem 13, we can get that $S_{\bar{\xi}}(\cdot)$ is l.s.c. at $\mu_{0}$.

Theorem 15. Let $\mu_{0} \in M$ be a considered point. Suppose that the following conditions are satisfied:

(i) $A(\cdot)$ is continuous with nonempty compact convex values at $\mu_{0}$;

(ii) $f(\cdot, \cdot, \cdot)$ is continuous on $B \times B \times M$.

Then, $S(\cdot)$ is u.s.c. at $\mu_{0}$.

Proof. Suppose the contrary that $S(\cdot)$ is not upper semicontinuous at $\mu_{0}$. Then, there exist an open neighborhood $U$ of $S\left(\mu_{0}\right)$ and a net $\left\{\mu_{\alpha}: \alpha \in \Lambda\right\}$ converging to $\mu_{0}$ such that

$$
S\left(\mu_{\alpha}\right) \nsubseteq U, \quad \forall \alpha \in \Lambda
$$

Then there exists some $x_{\alpha} \in S\left(\mu_{\alpha}\right)$ such that

$$
x_{\alpha} \notin U, \quad \forall \alpha \in \Lambda \text {. }
$$

Since $x_{\alpha} \in S\left(\mu_{\alpha}\right)$, we have $x_{\alpha} \in A\left(\mu_{\alpha}\right)$. By the assumption, $A(\cdot)$ is u.s.c. with compact valued at $\mu_{0}$, then we have that there exists subnet $\left\{x_{\alpha_{\beta}}\right\}$ such that $x_{\alpha_{\beta}} \rightarrow x^{*}$.

We will show that $x^{*} \in S\left(\mu_{0}\right)$; suppose the contrary that $x^{*} \notin S\left(\mu_{0}\right)$. Then there exists $y^{*} \in A\left(\mu_{0}\right)$ such that

$$
f\left(x^{*}, y^{*}, \mu_{0}\right) \in-\operatorname{int} C \text {. }
$$


Since $A(\cdot)$ is l.s.c. at $\mu_{0}$ and $y^{*} \in A\left(\mu_{0}\right)$ and $\mu_{\alpha} \rightarrow \mu_{0}$, we have that there exists $y_{\alpha} \in A\left(\mu_{\alpha}\right)$ such that $y_{\alpha} \rightarrow y^{*}$. It follows from $y_{\alpha} \in A\left(\mu_{\alpha}\right)$ that

$$
f\left(x_{\alpha}, y_{\alpha}, \mu_{\alpha}\right) \notin-\operatorname{int} C \quad \forall \alpha .
$$

By (ii) it implies that $f\left(x^{*}, y^{*}, \mu_{0}\right) \notin-\operatorname{int} C$, which leads to a contradiction with (30). Hence, we have $x^{*} \in S\left(\mu_{0}\right) \subseteq U$.

Since $x_{\alpha} \rightarrow x^{*}$ and $U$ is an open set, there exists some $\alpha_{0} \in \Lambda$ such that

$$
x_{\alpha} \in U, \quad \forall \alpha \geq \alpha_{0}
$$

which leads to contradiction with (29). Thus $S(\cdot)$ is u.s.c. at $\mu_{0}$.

The following theorem is directly obtained from Theorems 13 and 15.

Theorem 16. Let $\mu_{0} \in M$ be a considered point. Suppose that the following conditions are satisfied:

(i) $A(\cdot)$ is continuous with nonempty compact convex values at $\mu_{0}$;

(ii) $f(\cdot, \cdot, \cdot)$ is continuous on $B \times B \times M$;

(iii) for each $x \in A\left(\mu_{0}\right), f\left(x, \cdot, \mu_{0}\right)$ is $C$-strictly convex on $A\left(\mu_{0}\right)$;

(iv) for each $\mu \in M$ and $x \in A(\mu), f(x, \cdot, \mu)$ is C-convexlike on $A(\mu)$;

(v) $f\left(\cdot, \cdot, \mu_{0}\right)$ is monotone on $A\left(\mu_{0}\right)$.

Then, $S(\cdot)$ is continuous at $\mu_{0}$.

\section{Vector Optimization Problem}

Since the parametric weak vector equilibrium problem (PWVEP) contains the parametric weak vector optimization problems, we can derive from Theorem 17 direct consequences. We denote the ordering induced by $C$ as follows:

$$
\begin{aligned}
& x \leq y \quad \text { iff } y-x \in C ; \\
& x<y \quad \text { iff } y-x \in \operatorname{int} C .
\end{aligned}
$$

The ordering $\geq$ and the ordering $>$ are defined similarly. Let $g: X \times M \rightarrow Y$ be a vector-valued mapping. For each $\mu \epsilon$ $M$, consider the problem of parametric weak optimization problem (PWVOP) finding $x_{0} \in A(\mu)$ such that

$$
g(y, \mu)-g\left(x_{0}, \mu\right) \notin-\operatorname{int} C, \quad \forall y \in A(\mu) \text {. }
$$

Setting $f(x, y, \mu)=g(y, \mu)-g(x, \mu)$, PWVEP becomes a special case of PWVOP.

For each $\mu \in M$, the efficient solution set of (34) is denoted by

$$
\begin{aligned}
S^{\mathrm{OP}}(\mu):=\{ & x \in A(\mu): g(y, \mu) \\
& \left.-g\left(x_{0}, \mu\right) \notin-\operatorname{int} C, \forall y \in A(\mu)\right\} .
\end{aligned}
$$

The $\xi$-efficient solution set of (34) is

$$
\begin{aligned}
S_{\xi}^{\mathrm{OP}}(\mu):=\{x & \in A(\mu): \xi(g(y, \mu)) \\
& \left.\geq \xi\left(g\left(x_{0}, \mu\right)\right), \forall y \in A(\mu)\right\} .
\end{aligned}
$$

We directly obtain the following theorem from Theorem 16 .

Theorem 17. Let $\mu_{0} \in M$ be a considered point. Suppose that the following conditions are satisfied:

(i) $A(\cdot)$ is continuous with nonempty compact convex values at $\mu_{0}$;

(ii) $g(\cdot, \cdot)$ is continuous on $B \times M$;

(iii) for each $x \in A\left(\mu_{0}\right), g\left(\cdot, \mu_{0}\right)$ is C-strictly convex on $A\left(\mu_{0}\right)$;

(iv) for each $\mu \in M$ and $x \in A(\mu), g(\cdot, \mu)$ is $C$-convexlike on $A(\mu)$.

Then, $S(\cdot)$ is continuous at $\mu_{0}$.

The following example illustrates that the strict convexity cannot be dropped.

Example 18. Let $X=Z=\mathbb{R}, Y=\mathbb{R}, C=[0,+\infty), M=[0,1]$ be a subset of $Z$. Let $\mu_{0}=0 \in M$ be a considered point for PWVOP. Let $A: M \rightarrow X$ be a mapping defined by $A(\mu)=$ $[0,1]$ and let $g: X \times M \rightarrow \mathbb{R}$ be a mapping defined by

$$
g(x, \mu)=(\mu x, \mu x) \text {. }
$$

It is clear that $g$ does not satisfy $C$-strictly convex on $A\left(\mu_{0}\right)$. It follows from direct computation that

$$
S^{\mathrm{OP}}(\mu)= \begin{cases}{[0,1],} & \text { if } \mu=0, \\ \{0\}, & \text { if } \mu \in(0,1]\end{cases}
$$

Clearly, we see that $S(\cdot)$ is not l.s.c. at $\mu_{0}$. Hence, the assumed strict convexity of $g$ is essential.

\section{Conclusions}

In this paper, we study the lower semicontinuity of the set of efficient solutions for parametric weak vector equilibrium problems when the $\xi$-efficient solution set is a general set. Moreover, our theorems can apply for vector optimization problems.

\section{Conflict of Interests}

The authors declare that they have no competing interests.

\section{Authors' Contribution}

All authors read and approved the final paper. 


\section{Acknowledgments}

Pakkapon Preechasilp was supported by Pibulsongkram Rajabhat University. Rabian Wangkeeree was partially supported by the Thailand Research Fund, Grant no. RSA5780003. The authors would like to thank the referees for reading this paper carefully, providing valuable suggestions and comments on the original version of this paper.

\section{References}

[1] B. Chen and N.-J. Huang, "Continuity of the solution mapping to parametric generalized vector equilibrium problems," Journal of Global Optimization, vol. 56, no. 4, pp. 1515-1528, 2013.

[2] Q. H. Ansari, "Vector equilibrium problems and vector variational inequalities," in Vector Variational Inequalities and Vector Equilibria, F. Giannessi, Ed., vol. 38 of Nonconvex Optimization and Its Applications, pp. 1-15, Kluwer, Dordrecht, The Netherlands, 2000.

[3] X. B. Li and S. J. Li, "Existence of solutions for generalized vector quasi-equilibrium problems," Optimization Letters, vol. 4, no. 1, pp. 17-28, 2010.

[4] X. H. Gong, "Continuity of the solution set to parametric weak vector equilibrium problems," Journal of Optimization Theory and Applications, vol. 139, no. 1, pp. 35-46, 2008.

[5] Y. H. Cheng and D. L. Zhu, "Global stability results for the weak vector variational inequality," Journal of Global Optimization, vol. 32, no. 4, pp. 543-550, 2005.

[6] X. H. Gong and J. C. Yao, "Lower semicontinuity of the set of efficient solutions for generalized systems," Journal of Optimization Theory and Applications, vol. 138, no. 2, pp. 197205, 2008.

[7] S. Xu and S.-J. Li, "A new proof approach to lower semicontinuity for parametric vector equilibrium problems," Optimization Letters, vol. 3, no. 3, pp. 453-459, 2009.

[8] C. R. Chen and S. J. Li, "On the solution continuity of parametric generalized systems," Pacific Journal of Optimization, vol. 6, no. 1, pp. 141-151, 2010.

[9] Z. Y. Peng and S. S. Chang, "On the lower semicontinuity of the set of efficient solutions to parametric generalized systems," Optimization Letters, vol. 8, no. 1, pp. 159-169, 2014.

[10] B. T. Kien, "On the lower semicontinuity of optimal solution sets," Optimization, vol. 54, no. 2, pp. 123-130, 2005.

[11] J.-P. Aubin and I. Ekeland, Applied Nonlinear Analysis, John Wiley \& Sons, New York, NY, USA, 1984.

[12] F. Ferro, "A minimax theorem for vector-valued functions," Journal of Optimization Theory and Applications, vol. 60, no. 1, pp. 19-31, 1989.

[13] X. H. Gong, "Efficiency and Henig efficiency for vector equilibrium problems," Journal of Optimization Theory and Applications, vol. 108, no. 1, pp. 139-154, 2001.

[14] C. Berge, Topological Spaces, Oliver and Boyd, London, UK, 1963. 


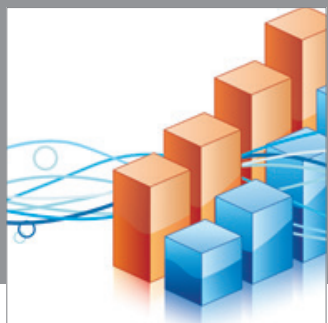

Advances in

Operations Research

mansans

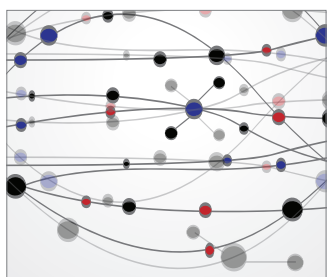

The Scientific World Journal
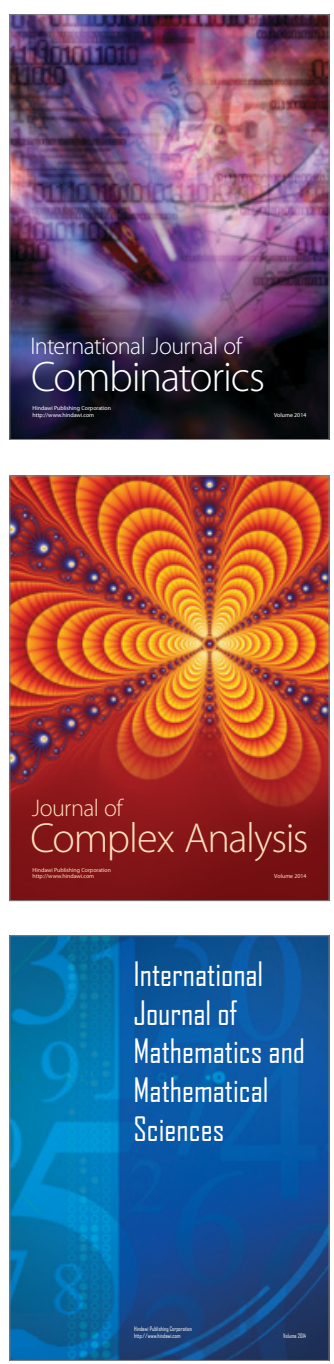
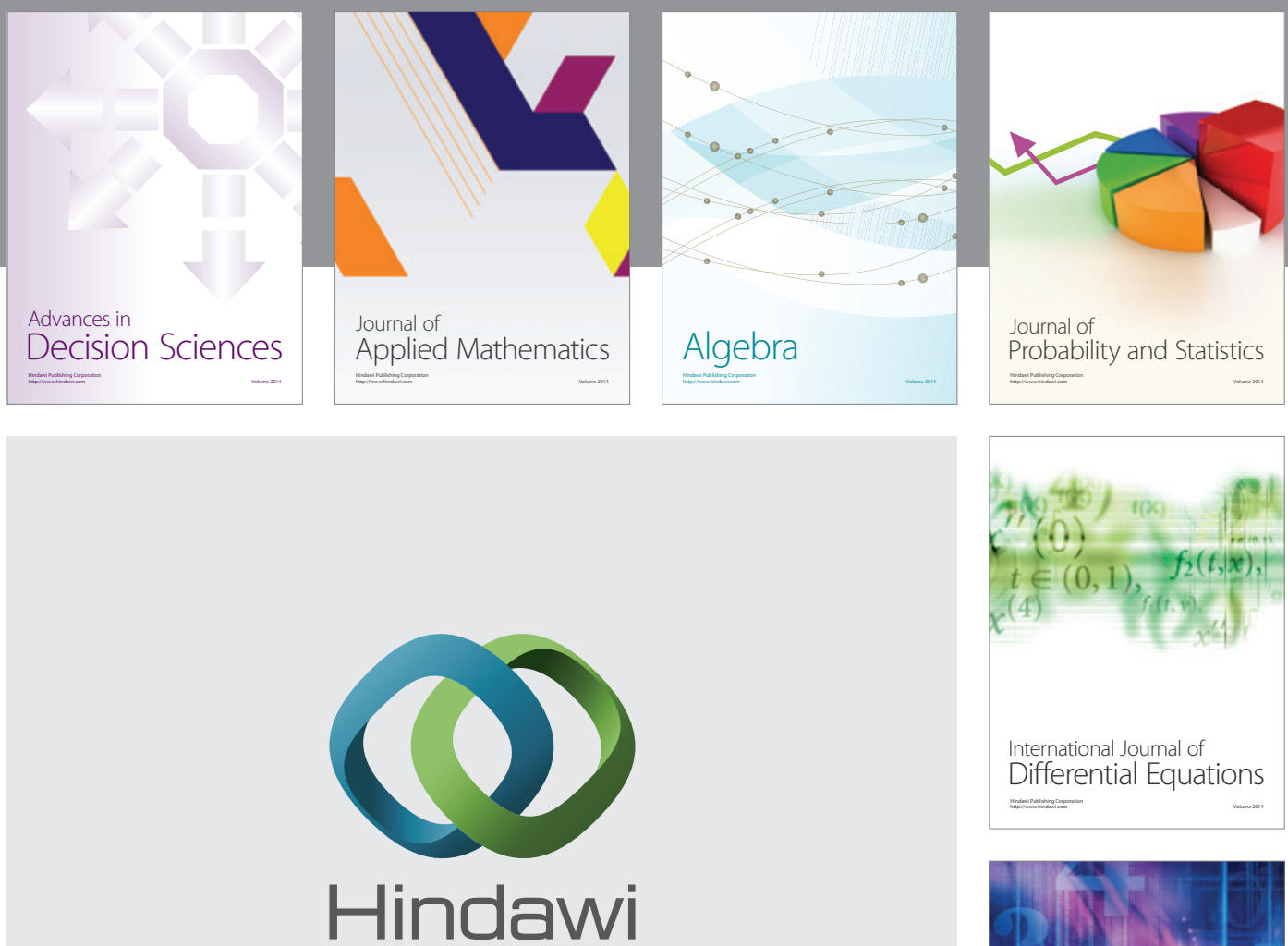

Submit your manuscripts at http://www.hindawi.com
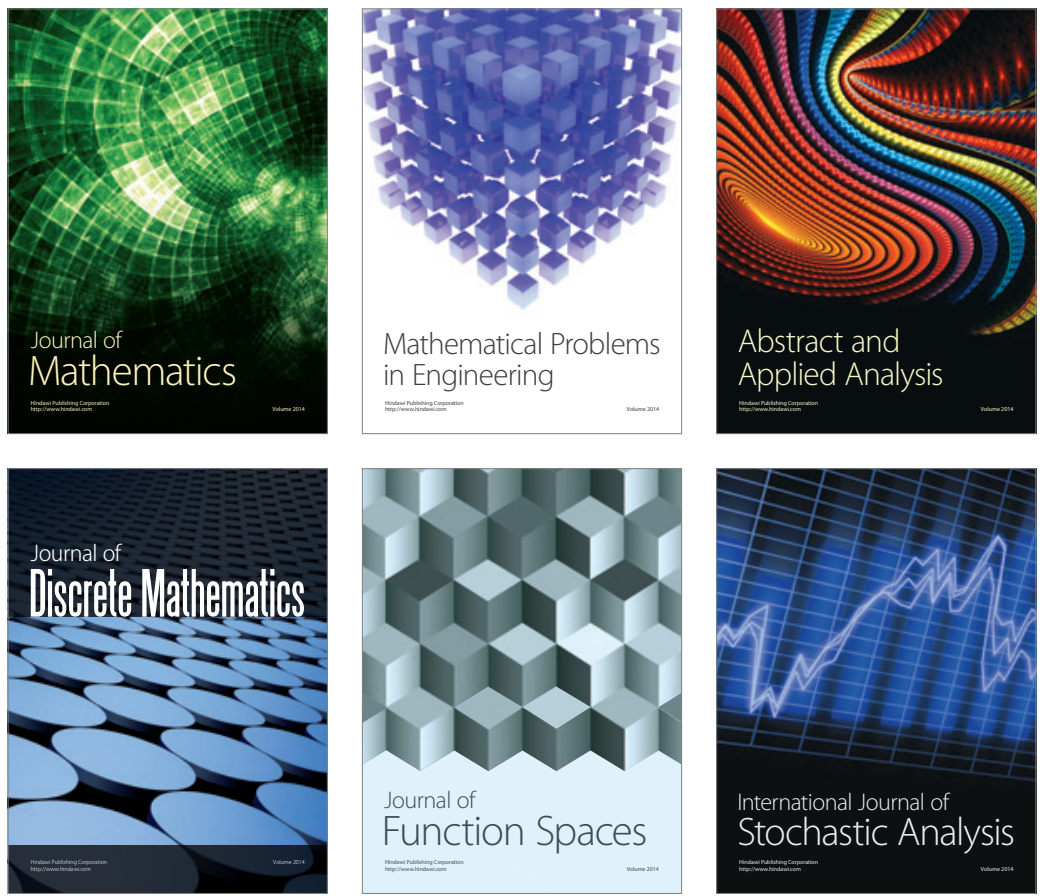

Journal of

Function Spaces

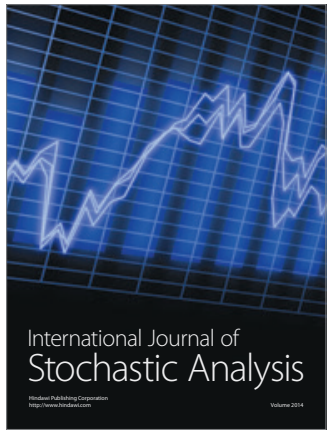

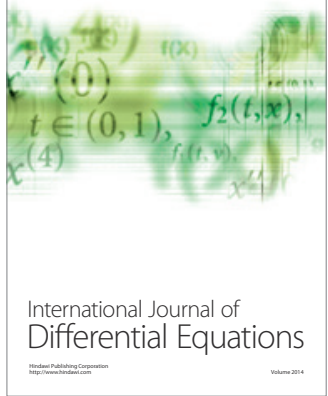
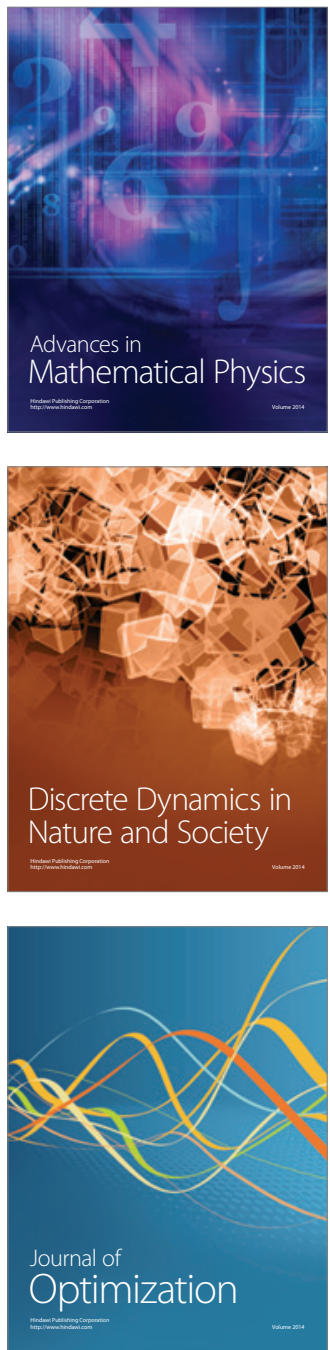